Lints, T. (2010). Diversity issues in adaptive modular systems. In 4th Annual IEEE Systems Conference, pages 207-210. IEEE.

T. Lints, "Diversity issues in adaptive modular systems," in 4th Annual IEEE Systems Conference, pp. 207-210, IEEE, 2010.

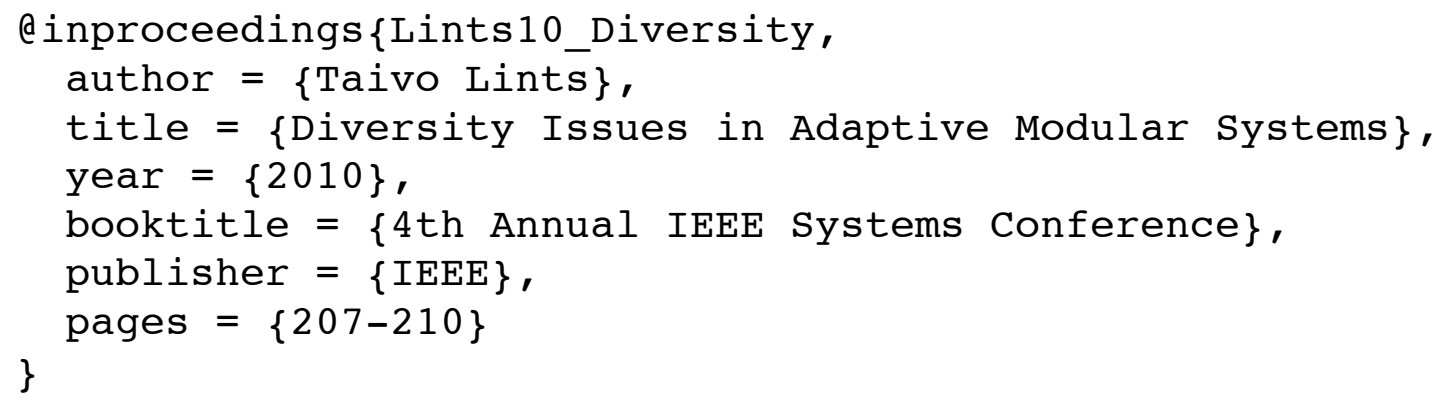




\title{
Diversity Issues in Adaptive Modular Systems
}

\author{
Taivo Lints \\ Research Laboratory for Proactive Technologies \\ Tallinn University of Technology \\ Ehitajate tee 5, 19086 Tallinn, Estonia \\ Email: taivo@taivo.net
}

\begin{abstract}
The majority of modular systems are composed of nonidentical components and are thus internally diverse. The paper points out and briefly discusses some of the main issues related to this diversity, especially in the context of adaptive systems: keeping the diversity relevant to the problem, maintaining an appropriate level of it, and avoiding or solving the conflicts between different components.
\end{abstract}

\section{INTRODUCTION}

If we subtract the fairly small subset of systems composed of fully identical elements, the rest of the modular systems we are left with are by definition internally diverse. And diversity can considerably aid collective problem solving (which can be taken to include all kinds of goal-directed behaviors) because different viewpoints and capabilities of the individuals complement each other. However, as pointed out in [1]: "difference does not magically translate into benefits" but requires some conditions to be met first. And, additionally, assuring that the type and level of diversity in the system move to the region of profitability and also stay there is not always trivial. Thus, for a system to achieve its goals efficiently, the diversity should usually be managed in some way, implicit or explicit. Among the most important points to pay attention to when doing it are:

- keeping the diversity relevant to the problem, as opposed to trying to have as much of it as possible in all aspects,

- maintaining an appropriate level of diversity,

- and solving the conflicts between different components, fostering openness and cooperation.

The majority of the ideas and suggestions in the following paper are drawn from the research literature on human organizations. This naturally means that the given collection seems to be most applicable to those systems where the main components are indeed humans. However, even though this is most probably true and it is perfectly fine to use the article as a supportive set of notes for designing and managing human teams, the key intent of the paper is to provide inspiration for interdisciplinary idea transfer. Therefore, the readers are advised to creatively consider the applicability of the given suggestions to their own systems of interest, no matter if

This work was supported in part by Research Laboratory for Proactive Technologies in Tallinn University of Technology, Department of Computer Control in Tallinn University of Technology, Estonian Doctoral School in ICT, Estonian Information Technology Foundation, and Estonian Ministry of Education and Research (grant SF0140113As08). the components of those are humans, human teams, large organizations, hardware units, software modules, nonhuman biological entities, mixed techno-social systems, or anything else.

\section{Keeping the Diversity Relevant to the Problem}

First of all, it is advisable to keep the diversity relevant to the problem(s) faced by the system instead of just trying to maximize it at any cost [1, page xxix]. Typically this would be too obvious even to mention, but for the last few decades the term diversity has been rather sensitive politically and ethically in the context of human systems, where it has so far been quite narrowly centered (at least within the wider audience) on race, gender and identity, and any questioning of the superiority of it over homogeneity can cause quite painful reactions. The latter, in turn, may have lead many people to automatically associate any diversity with advantageousness and to forget checking the validity of that link in their particular context.

But even in human systems the need for a more thorough and detailed approach is becoming evident, because if the preferences of the individuals are too diverse, especially the fundamental preferences that tend to be outside the scope of negotiations and compromises, then any improvement for one person would make matters worse for the others, or, in the case of resource sharing: "More diverse fundamental preferences should result in fewer resources allocated to collective goods and projects - things that everyone can use. The collective pie has to be sliced into many thin slices consumed by individuals." [1, page 282]. In nonhuman components the elements corresponding to fundamental preferences might be the top level goals and purposes of those components, and maybe also some lower-level behaviors and functionalities that are unchangeable ("hardwired") and impossible to override or deactivate.

Therefore, instead of the fundamental preferences, the focus of diversification should be on perspectives, heuristics, predictive models, methods of and instruments for achieving something, and the like. That is, the subjects of diversity should first and foremost be those elements, tools and functionalities that, when combined, provide additional capabilities instead of running into deadlocks and blocking even the existing few options or just being too different for any useful complementary combination to occur. 
Additional possible problems stemming from excessive and unmanaged diversity are listed in [2]:

...several other studies examining the impact of diversity at an individual level have shown that when compared to similar individuals, people who are different (dissimilar individuals) have less attraction and trust in peers (Chatopadhyay, 1999), less frequent communication (Zenger \& Lawrence, 1989), lower group commitment (Tsui, Egan, \& O'Reilly, 1992), lower task contributions (Kirchmeyer, 1993; Kirchmeyer \& Cohen, 1992), lower perceptions of organizational fairness and inclusiveness (MorBarak, Cherin, \& Berkman, 1998). At a group level, similar results have been found. Compared to homogeneous groups, heterogeneous groups are found to have reduced cohesiveness (Terborg, Castore, \& DeNinno, 1976; Harrison et al. 1998), more conflicts and misunderstandings (Jehn, Chadwick, \& Thatcher, 1997) which, in turn, lowers members' satisfaction, decreases cooperation (Chatman \& Flynn, 2001; Chatman \& Sparato, 2005), and increases turnover (Jackson, Brett, Sessa, Cooper, Julin, \& Peyronnin, 1991; Jackson et al., 1991).

Thus, considerable care should be taken to not overdo the diversification "just because it was said to be great", and if the problems the system is facing are straightforward with known clear unquestionably best solutions, then the system may actually need no diversity at all (at least with regard to these specific tasks).

But by no means should this long list of potential problems be taken as a suggestion to dismiss diversity altogether. When the pitfalls are successfully avoided and supportive mechanisms in place, the diversity can provide real and provable benefits by allowing systems to find better solutions to their problems, increasing their stability and / or adaptivity, and so forth (see, e.g., [1]-[3]). In a kind of a meta-level approach, especially in larger systems (and particularly in systemsof-systems) it might even be useful to have a diversity of diversities, with some individuals or teams being diverse and others more specialized and / or homogeneous [1, page 173], thus simultaneously capitalizing on the specific benefits of both options.

\section{MAintAining THE LEVEL OF DiVERsity}

If the components are neither changing much nor being excluded from, or included to, the system based on some selection criteria, the amount of initial diversity is typically preserved over time, but the adaptive capacity of the system might also be fairly limited. In systems with no such limitations, however, the drive towards better performance, or some other factors, can easily result in the decrease of diversity. This is not necessarily bad, and in fact the removal of less fit elements is the very basis of most adaptational processes, but the problem arises if the removal and streamlining is shortsightedly taken too far and not enough capacity is left to cope with various currently not encountered disturbances that however may hit in the future.

There seem to be no quick fixes for that "efficiency deficiency". In general, it would be necessary to find some balance between getting higher adaptivity plus enough robustness and having a system with very high efficiency with regard to current conditions. This is certainly not an easy task even for a single isolated system, but can be exceptionally problematic in a competitive ecosystem situation - trying to achieve longterm success usually requires a degree of protection against unexpected disturbances, which, however, typically somewhat diminishes current efficiency, which in turn may allow some shortsighted rivals (who maximize for the moment and do not care about the future) to drive the more cautious system into extinction. The high likelihood of that shortsighted rival being knocked out of existence shortly thereafter by external events is of little consolation to the cautious one.

What might help to some extent is trying to achieve maximal possible adaptivity with minimal extra machinery. That is, to be able in most circumstances to achieve a level of efficiency close enough to the shortsighted rivals so as to avoid extinction (or to find some other competitive survival strategy), but when situation changes then modify itself in order to match the new situation rapidly enough to keep the efficiency gap sufficiently small with regard to the new upcoming batch of shortsighted competitors. The means for such behavior include changeable levels of diversity, as well as of integration, specialization, and modularity. But even then the limited-time-perspective competitors would in most relatively stationary cases still be able to achieve some superiority in efficiency. A more promising option would be to make use of the internal diversity in such a way that the system can exploit a broader range of resources than any single more specialized and homogeneous competitor, and thus compensate for the lack of depth with breadth.

As of the mechanisms of avoiding the loss of diversity, one hint for a potential solution can be found from the studies of natural ecosystems, where the diversity maintenance is considered to require the existence of flux or variability in the ecosystem together with populations capable of differentially exploiting this flux or variability [3, summarizing the study in [4]]. The required variability might be realized as, for example, spatial heterogeneity of the environment or temporal variations of miscellaneous conditions and parameters. While in natural ecosystems these can be expected to occur, well, naturally, in artificial systems it may be necessary to deliberately add mechanisms that, depending on the requirements, either keep generating the variability continuously or add new variations only occasionally, when really needed.

Additionally, if the system has an ability to allow or even promote local behavioral exploration (i.e., the generation of variability, diversity, and new solutions at the component or subsystem level) and to spread the found strategies (preferably the successful ones, but in practice also others) all over the system, it should also keep an eye on the diversity level that might decrease too much if the spreading mechanism is 
"overly efficient". In systems consisting of components with advanced cognitive capabilities the problem is often referred to as groupthink. The most straightforward method of avoiding harmful groupthink would be to limit the spreading of ideas and strategies within the system, but that would also cut down the propagation of useful strategies and thus keep the system from reaching the level of efficiency it could otherwise achieve. Luckily, several other techniques with less negative side effects have been suggested, too (again, mainly for human systems, but the ideas can give some inspiration for other application areas as well):

- Encouraging the individuals "to make their own assessments and, where appropriate, to retain their private views [...], but also to constantly challenge their own thinking and to be prepared to look for both confirming and contradictory evidence" [5].

- Challenging "personal and organizational assumptions, beliefs, and values to determine their relevancy" for both the current situation and for the future [6].

- Questioning one's associates and arguing with them even if the ideas and viewpoints are still in development, i.e., not waiting until the paradigms get established and fixed, even though it may occasionally feel inappropriate to critique ideas that are still under construction [7]. But, surely, the criticizing should not be overdone into destruction of exploration.

- Higher level managers, if such exist in the system, could foster variety "by encouraging varied interests and points of view in organizational members; by generating lots of data; by calculating [i.e., providing] wide exposure of [lower-level] managers to the organization's environment; and by requiring the initial processing of data in an unstructured, equivocal manner" [8].

So, broadly speaking, the goal of the system designer or manager (or, possibly, of the system itself) would be to determine (or at least estimate), for all the important functionalities and for any given situation, the (near-)optimal levels of diversity of the relevant aspects of the system, and then increase or decrease the diversity accordingly. Except, of course, if the potential benefits gained from managing the levels of diversity do not justify the costs of doing it.

\section{AVOIding And Solving Conflicts}

While identical components have similar needs and may thus fall into conflict with each other mainly over their access to some limited resource, diverse components are, additionally, likely to clash due to different opinions on how to solve some problem, on how to make the best use of limited resources, on priorities and even the goals of the system. Thus diversity management generally needs some mechanisms for solving the conflicts, as well as for fostering cooperation and openness to differences. In this regard, an ideal system would consist of elements that have high levels of tolerance and courtesy, and that are willing to compromise. The value system should encourage a culture of learning, thinking and discussion, of free speech and pluralism. To achieve this, the individuals should feel safe to express their opinions and to engage in open communication, and they should know that potential conflicts will be solved in a constructive manner. Otherwise they might prefer to keep the number of conflicts low via not disclosing their points of view, which in turn may decrease their, and the system's overall, performance due to the useful information and ideas not being communicated within the system [2].

Speaking more operationally, the techniques for arriving to reasonable decisions despite diverse ideas include:

- creating an environment that "provides a sense of confidence in which no member feels he/she will be rejected, embarrassed, or punished for speaking up" [2];

- having the components emphasize their interests and goals instead of positions and methods, because even if the initially considered ways of achieving each party's goals are mutually exclusive, there may be other less obvious ways that are not [1, page 350$]$;

- identifying areas of consensus and points of difference between (mental) models [5], as opposed to automatically considering all less than fully compatible models to be fully incompatible;

- the use of simulation, probability, and judgement heuristics (nonprobabilistic methods that make use of consensus between knowledgeable people) [9, page 103];

- the use of polling and voting schemes;

- information markets that, unlike polls, can create both an incentive to be correct and an incentive to be diverse, e.g., payoff to each market participant being proportional to how correct / useful was the information he/she/it provided and inversely proportional to how many others provided similar information, just like in horse race betting [1, pages 233-234];

- and many more.

Additionally, special mediator components could be used that arbitrate among other elements, trying "to minimize conflict, interferences and frictions; and to maximize cooperation and synergy" [10]. The description of (efficient) organizational sensemaking from [11] (as cited in [7]) is also quite educative for diversity management: "coordination of action over alignment of cognitions, mutual respect over agreement, trust over empathy, diversity over homogeneity, loose over tight coupling, and strategic communication over unrestricted candor".

While a lot of the aforementioned conflict alleviation methods are inspired by (but by no means restricted to!) human group behavior, it is important to notice that there also exist further possibilities which are generally considered unsuitable in modern human teams but are perfectly valid in other contexts. For example, as described in [10], instead of compromises and agreements there could be imposition (forcing the opposing components to change despite their disapproval) or even eradication (elimination of some opposing components by others). The same source [10] also lists an intermediate option between the forced and "civilized" ones, that of apoptosis, which is common in biological multicellular 
organisms and denotes the situation where a component (cell) that for some reason is not acceptable to the system anymore self-destructs in a controlled manner for the benefit of the system as a whole. Such approaches, however, tend to decrease the diversity and should thus be used with care (i.e., balanced with the creation of new, different, variations, if and when deemed necessary).

\section{CONCLUSION}

Diversity can be a beneficial property as long as it is wisely managed - kept relevant to the problem, maintained at an appropriate level, and held from degrading into unconstructive conflicts and deadlocks. But, as always, nothing is universally good for every purpose and in every situation, and so it is with diversity, too. Thus, a thorough analysis of how much and which kind of diversity, as well as exactly how and where, to apply would be advisable for each specific practical case where the potential benefits might justify the costs of that analysis. And in order to do it effectively, at least as long as no comprehensive overarching integrative theory and guidelines of diversity have been put together, it remains highly helpful to keep an eye on all the different disciplines that deal with problems related to the topic, and try to foster idea transfer between them.

\section{ACKNOWLEDGMENT}

Thanks to Leo Mõtus for providing me the possibility to do highly unconstrained research.

\section{REFERENCES}

[1] S. E. Page, The Difference: How the Power of Diversity Creates Better Groups, Firms, Schools, and Societies. Princeton University Press, 2007.

[2] M. Élène Roberge and R. van Dick, "Recognizing the benefits of diversity: When and how does diversity increase group performance?" Human Resource Management Review, 2009, article in press.

[3] K. S. McCann, "The diversity-stability debate," Nature, vol. 405, pp. 228-233, May 2000.

[4] P. Chesson and N. Huntly, "The roles of harsh and fluctuating conditions in the dynamics of ecological communities," The American Naturalist, vol. 150, no. 5, pp. 519-553, November 1997.

[5] A.-M. Grisogono and A. Ryan, "Operationalising adaptive campaigning," in International Command and Control Research and Technology Symposium, 12th ICCRTS, 2007.

[6] U.S. Army War College, "Strategic Thinking (ST), first course of the academic year," http://www.carlisle.army.mil/USAWC/dclm/fst.htm.

[7] K. E. Weick, "Managing the unexpected: Complexity as distributed sensemaking," in Uncertainty and Surprise in Complex Systems, R. R. McDaniel Jr. and D. J. Driebe, Eds. Springer, 2005, pp. 51-65.

[8] J. J. Sullivan and I. Nonaka, "The application of organizational learning theory to Japanese and American management," Journal of International Business Studies, vol. 17, no. 3, pp. 127-147, 1986.

[9] E. F. Moran, Human Adaptability: An Introduction to Ecological Anthropology, 2nd ed. Westview Press, 2000.

[10] C. Gershenson, "Design and control of self-organizing systems," Doctoral dissertation. Vrije Universiteit Brussel, 2007.

[11] E. M. Eisenberg, "Jamming: Transcendence through organizing," Communication Research, vol. 17, no. 2, pp. 139-164, April 1990. 